\title{
The effect of laparoscopy and laparotomy on hemostasis in experimental colorectal cancer surgery in rats
}

\author{
Eyüp Murat Yılmaz ${ }^{1}$, Erdem Barış Cartı ${ }^{1}$, Çiğdem Yenisey², Mahir Kırnap", Muhammed Çağrı Coşkun¹, Ahmet \\ Ender Demirkıran ${ }^{1}$, Nesibe Kahraman Çetin ${ }^{3}$
}

${ }^{1}$ General Surgery Department, Aydın Adnan Menderes Üniversity, Aydin, Turkey

${ }^{2}$ Biochemistry Department, Aydin Adnan Menderes Üniversity, Aydin, Turkey

${ }^{3}$ Pathology Department, Aydın Adnan Menderes Üniversity, Aydin, Turkey

Received: 2020-09-11.

Accepted: 2020-10-16

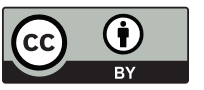

This work is licensed under a

Creative Commons Attribution 4.0 International License

J Clin Med Kaz 2020; 6(60):83-87

Corresponding author:

Eyüp Murat Yılmaz.

E-mail: drmyilmaz80@gmail.com;

ORCID: 0000-0002-0808-5067

\section{Abstract}

Background: Nowadays, it is claimed that advanced laparoscopic methods are minimally invasive, provide quick recovery of normal functions in patients and cause less stress in the organism compared to similar open surgery techniques. There is only limited research on 8-OHdG and 8-OHG levels, which are the markers of oxidative stress in both laparoscopic and open surgery. In this experimental study, we investigated the stress level caused by the laparotomic and laparoscopic colon resections in rats with induced colorectal cancer model.

Material and methods: In this study, colon cancer was induced with subcutaneous dimethylhydrazine $(\mathrm{DMH})(20 \mathrm{mg} / \mathrm{kg})$ in 30 SpragueDawley male rats. After 12 weeks, rats were divided into three groups: Group 1 (control group; $n=10$ ), Group 2 (laparotomy group; $n=10$ ), and Group 2 (laparoscopy group; $n=10$ ). Afterward, blood and lung tissue samples were obtained, and 3-NT, 4-HNE, OHdG, PGF2, and NO levels were measured.

Results: There was a statistically significant difference between the groups in terms of 3-NT (ng/ml), which is a biomarker of oxidative stress $(\mathrm{p}<0.01)$. After obtaining blood samples from rats, DNA and RNA were isolated, and a statistically significant difference was found between the laparotomy and laparoscopy groups in terms of 8-OHdG and 8-OHG molecules $(p<0.01)$.

Conclusion: Laparoscopy, which initiated a new era in the scientific field, provided various advantages over laparotomy and widely used in colorectal surgery. In addition, it was demonstrated that laparoscopic surgery caused less oxidative stress, had advantageous effects on the antioxidant defense system, and thus superior to laparotomy.

Keywords: laparoscopic surgery, colorectal cancer, laparotomy, 3-NT, 8-OHdG

\section{Introduction}

Hemostasis is essential for life. However, this complex and dynamic balance can be threatened by many exogenous or endogenous factors. Stress is defined as a factor, which may affect hemostasis and is related to the neuroendocrine and cellular changes in both central and peripheral nervous systems and depends on the adaptive response of the organism. Surgical stress causes a response in the peripheral organs, the hypothalamicpituitary-adrenal axis (HPA), sympathetic nervous system along with the renin-angiotensin axis, and it is well known that these responses are of vital importance. The surgical wound can impair hemostasis and increase bleeding risk depending on the size of the wound. Nowadays, laparoscopic surgery is preferred for the diagnosis and treatment of many disorders. Authors suggest that laparoscopy is a minimally invasive method, causes less psychological disturbance and less stress in the organism compared to open surgery and patients regain normal functions faster $[1,2]$.

We screened the studies focused on oxidative stress in laparoscopic and open surgery and the metaanalysis we found showed that all studies had been conducted with cholecystectomy patients. We detected only one study focused on colorectal tumor surgery [3], which was conducted on 60 patients. To the best of our 
knowledge, there is no study in the literature focused on DNA and RNA samples isolated for the assessment of 8-OHdG and 8-OHG levels, which are the markers of oxidative stress caused by laparoscopic and open surgery in patients with colorectal cancer. Furthermore, we did also not find any experimental study comparing the relationship of the impaired integrity of membranes, 8-iso-prostaglandin F2 $\alpha$ (a determinant of lipid peroxidation), protein carbonyl content (PCC) (an indicator used for the determination of the 4-HNE (4-hydroxynonenal) proteins that are an indicator of the lipid peroxidation), 3-nitrotyrosine (an indicator of nitrogen radicals of proteins and their damage), NO (nitric oxide) (the most important molecule of the oxidative burst that emerges as a result of the iNOS enzyme triggered by proinflammatory cytokine TNF- $\alpha$ ), and MPO (myeloperoxidase) in leukocytes, which is one of the most important indicators of oxidative burst [4].

In this study, our objective was to compare the levels of antioxidant molecules superoxide dismutase (SOD) and catalase (CAT), which are determinants of oxidative stress, in rats with experimental colon cancer, which underwent laparotomic and laparoscopic colon resection.

\section{Material and methods}

The study was approved by the Animal Experiments Local Ethics Committee of Aydin Adnan Menderes University (No: $64583101 / 2017 / 061)$. A total of 30 Sprague-Dawley male rats with a mean weight of 350-400 g were included in the study. This study was scientifically supported as a Scientific Research Project by Aydın Adnan Menderes University (No: ADÜ BAP TPF-17052).

To determine the oxidative damage, the below-mentioned levels of the molecules were assessed in the blood samples of the rats.

3-nitrotyrosine: Nitric oxide plays an important role in several biological processes including cell proliferation, apoptosis, neurotoxicity, and re-modeling of the extracellular matrix. NO forms peroxynitrite through the reaction with superoxide radicals, which forms the remnants of nitrated tyrosine in the proteins. Thus, it can be suggested that nitrotyrosine is a marker of the peroxynitrite and consequently of the cellular damage and oxidative stress.

Protein carbonyl content (PCC): It has been demonstrated that proteins are carbonylated during oxidative damage. This determinant is a very reliable and stable marker of protein oxidation.

4-HNE (4-hydroxynonenal): This molecule is one of the most reliable biomarkers for the lipid peroxidation caused by oxidative stress. This compound is a more stable molecule compared to MDA, which is also a marker for all lipid peroxidation.

8-OHG RNA Damage: Following the reactions between RNA hydroxyl free radical $\left(-\mathrm{OH}^{\circ}\right.$.) and nitrogen species, (8-hydroxyguanosine $(8-\mathrm{OHG})$ is formed as a result of the interaction between guanine and these compounds.

8-OHdG DNA Damage: Following the reactions between DNA hydroxyl free radical $\left(-\mathrm{OH}^{\cdot}.\right)$ and nitrogen species, (8-hydroxydesoxyguanosine $(8-\mathrm{OHdG})$ is formed as a result of the interaction between guanine and these compounds.

8 -iso-Prostaglandin F2 $\alpha$ : This molecule is one of the most reliable markers of in vivo oxidative damage and plays an important role in the pathogenesis of diseases. It is produced by the in vivo peroxidation of arachidonic acid and causes vasoconstriction. It can be detected in plasma and excreted with urine.
Myeloperoxidase (MPO): MPO is the most reliable marker of the respiratory burst during oxidative damage. As respiratory burst occurs in leukocytes, we isolated leukocytes from blood and carried out an analysis.

Nitric Oxide (NO): NO has an important role in the oxidative burst, and its release is induced by pro-inflammatory cytokines TNF- $\alpha$ and IL- $\beta$. The analysis was performed with serum samples.

\section{Formation of colon cancer model}

All rats received a weekly subcutaneous injection (shoulder skin) of $20 \mathrm{mg} / \mathrm{kg}$ dimethylhydrazine (DMH), and they were fed with regular food and water and monitored in the experimental laboratory.

\section{Experiment groups}

Group 1 (control group, $\mathrm{n}=10$ ): Sacrifice of rats was planned following blood collection.

Group 2 (laparoscopy group, $n=10$ ): After the maximum insufflation of the abdomen with the Veress needle and laparoscopic tumor resection with scissors through a $1-\mathrm{cm}$ incision, the sacrifice of rats was planned following blood collection.

Group 3 (laparotomy group, $\mathrm{n}=10$ ): After the location and resection of the tumor through a laparotomic midline-incision, the sacrifice of rats was planned following blood collection.

After the completion of the study, all rats were sacrificed with intraperitoneal ketamine injection $(150 \mathrm{mg} / \mathrm{kg})$. However, one rat in the laparotomy group died due to the abdominal distension in the 12th week, so that this group was evaluated with 9 rats $(n=9)$. As distension started to develop in rats at the end of the 12th week, we decided to discontinue DMH injections and proceed to the resection phase. The abdominal region was shaved and cleaned with the antiseptic solution, while animals were under sedation.

Anesthesia was administered with intraperitoneal ketamine $(40 \mathrm{mg} / \mathrm{kg})$ and xylazine $(5 \mathrm{mg} / \mathrm{kg})$. During the procedure, rats were resuscitated with $1.5 \mathrm{cc}$ intraperitoneal saline solution. The interventions were carried out as planned. In the control group, the rats were sacrificed after the collection of blood samples. In the laparoscopy group, the abdomen of the rats was insufflated to the maximum level with a Veress needle and the tumor was resected laparoscopically with scissors and the resected loop was removed through a $1-\mathrm{cm}$ incision. Then, blood samples were obtained, and the rats were sacrificed. In the laparotomy group, after a midline-incision was made and the colonic tumor region was located, the tumor was resected and removed from the abdomen. Then, blood samples were obtained, and the rats were sacrificed. The macroscopic samples, which were obtained during laparotomy, were sent for pathological examination. The pathological examination revealed obstructive polypoid mass lesion with mucus loss and reactive changes (Figure 1). 3-NT, 4-HNE, OHdG, PGF2, and NO levels were determined in blood and tissue samples. The comparative analysis was performed with statistical methods between the groups for the measured parameters.

\section{Statistical analysis}

The data were analyzed with SPSS v.25 (IBM Corp. Released 2017. IBM SPSS Statistics for Windows, Version 25.0. Armonk, NY: IBM Corp.) software package. First, the descriptive statistics of the continuous variables were calculated. The descriptive data were expressed in mean \pm standard deviation 
Figure 1 - A Mass with loss of mucus

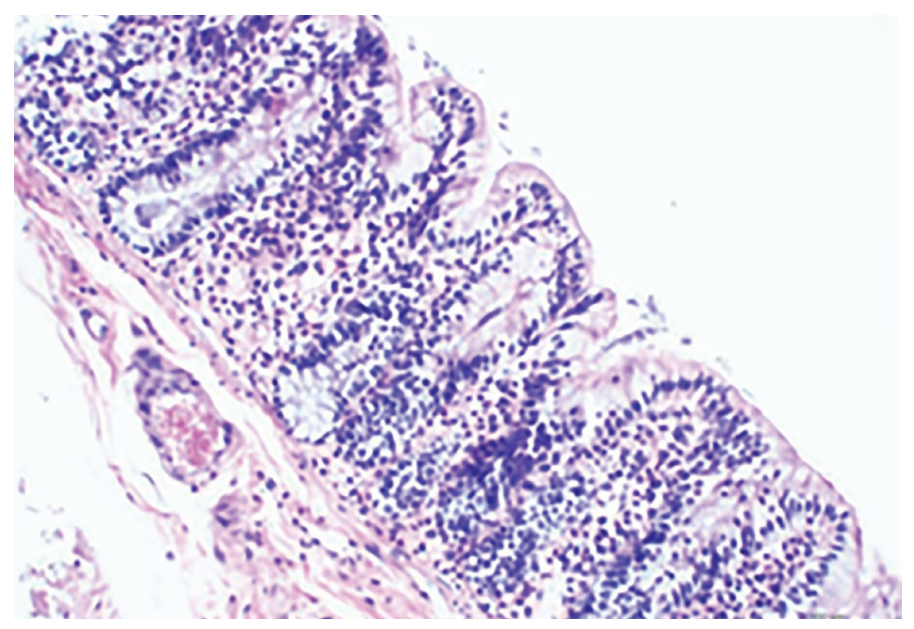

and frequency $(\%)$. The variables were evaluated with the Shapiro-Wilk and Levene tests after they were controlled for the requirements of the normal distribution and variance homogeneity. The Independent Paired Group t-Test (Student's t-test) was used for the comparison of two groups, and the Mann-Whitney $U$ test was used when the requirements were not met. The One-Way Variance Analysis and Multivariate Tukey HSD test (a multiple comparison test) were used for the comparison of three or more groups, and the Kruskal-Wallis test and Bonferroni-Dunn test (a multiple comparison test) were used if the requirements were not met. For all analyses, $\mathrm{p}<0.05$ was considered statistically significant.

\section{Table 1 Introductory Statistics on Variables}

\begin{tabular}{|c|c|c|c|c|c|}
\hline & $\mathrm{N}$ & Minimum & Maximum & Avarage & $\begin{array}{l}\text { Standard } \\
\text { deviation }\end{array}$ \\
\hline $\begin{array}{l}\text { 3-NT (ng/ } \\
\mathrm{mL})\end{array}$ & 29 & 7,602 & 120,941 & 48,66338 & 30,693790 \\
\hline $\begin{array}{l}\text { 4-HNE } \\
\text { (ng/mL) }\end{array}$ & 29 & 1,817 & 83,322 & 32,29921 & 24,799143 \\
\hline $\begin{array}{l}\text { OHdG } \\
\text { (ng/mL) }\end{array}$ & 29 & 6,205 & 102,813 & 52,70172 & 33,0861 \\
\hline $\begin{array}{l}\text { PGF2 (ng/ } \\
\mathrm{mL} \text { ) }\end{array}$ & 29 & 22,071 & 392,023 & 116,23200 & 88,1255 \\
\hline NO (M) & 29 & 12,112 & 79,304 & 30,90621 & 17,775628 \\
\hline Dil.Fx0,2 & 29 & 2,4224 & 15,8608 & 6,181241 & 3,5551255 \\
\hline Clear NO & 29 & 14,5344 & 95,1648 & 37,087448 & 21,33075 \\
\hline
\end{tabular}

Abbreviations: 3NT: 3 Nitrotirozin , 4-HNE :4-Hydroxynonenal, 8-OHdG:8-hydroxy-2' -deoxyguanosine

\section{Results}

In this study, we investigated the effects of laparoscopy and laparotomy on hemostasis in rats with induced colorectal cancer model. Following the isolation of DNA and RNA samples from blood, the oxidative damage in these samples was evaluated by detecting the $8-\mathrm{OHdG}$ and $8-\mathrm{OHG}$ molecules, respectively. In addition, we evaluated 8-iso-Prostaglandin F2 $\alpha$, 4-HNE, and protein carbonyl content, which are the markers of the lipid damage as a result of oxidative stress. We also evaluated serum NO and MPO, which emerges in leukocytes due to the respiratory burst and triggers inflammation. The measured levels were compared to antioxidants (CAT and SOD). 3-NT (ng/ml), 4-HNE (ng/ml), OHdG (ng/ml), PGF2 (ng/ml), NO (M), Dil. Fx0.2, Net NO $(\mu \mathrm{M})$ were used as descriptive data related to the intergroup variables (Table 1).

The comparison between the groups for 3-NT $(\mathrm{ng} / \mathrm{mL})$, 4-HNE (ng/mL), 8-OHdG (ng/mL), PGF2 (ng/mL) and Net NO $(\mu \mathrm{M})$ were performed with One-Way Variance Analysis (ANOVA) and Kruskal-Wallis test. The comparison of all three groups for 3-NT $(\mathrm{ng} / \mathrm{ml})$, which is a biomarker of oxidative stress, revealed a statistically significant difference $(\mathrm{p}<0.01)$. The mean value was lower in the control group compared to the laparotomy and laparoscopy groups. The highest mean values were in the laparotomy group. This finding indicated that the deterioration of hemostasis related to acute and chronic inflammation was most prominent in the laparotomy group. It was suggested that 4 -HNE played a key role in the cellular signal transduction through various ways extending from cellular cycle events to cellular adhesion processes. There was a statistically significant difference between the three groups in terms of $4-H N E(n g / m l)(p<0.01)$. The mean value of the control group was lower than the laparotomy and laparoscopy groups. The comparison of laparoscopy and laparotomy groups showed that the mean 4-HNE level was higher in the laparotomy group. 8-OHdG levels are widely used as a biomarker for the oxidative lesion, consequently for oxidative stress and carcinogenesis. The comparison of the three groups showed a statistically significant difference in terms of $8-\mathrm{OHdG}(\mathrm{ng} / \mathrm{mL})(\mathrm{p}<0.01)$. Although the lowest mean value was observed in the control group, it was also statistically lower in the laparoscopy group compared to the laparotomy group. Furthermore, there was a statistically significant difference between the three groups in terms of PGF2 $(\mathrm{ng} / \mathrm{ml})$ and Net NO $(\mu \mathrm{M})(\mathrm{p}<0.01)$. The mean values of these parameters were lowest in the control group compared to the other two groups (Table 2).

Table 2

Comparison of 3-NT (ng/mL), 4-HNE (ng/mL), 8OHdG (ng/mL), PGF2 (ng/mL) and Net NO $(\mu M)$ variables with three groups

\begin{tabular}{|c|c|c|c|c|c|}
\hline & Control & Open & Laparoscopic & \multirow[t]{2}{*}{ Test Statistics } & \multirow[t]{2}{*}{$\mathrm{p}$} \\
\hline & $n=10$ & $n=9$ & $\mathrm{n}=10$ & & \\
\hline 3-NT (ng/mL) & $\begin{array}{l}19,357,94 a \\
(7,60-29,75)\end{array}$ & $\begin{array}{l}82,3825,20 \mathrm{~b} \\
(53,32-120,94)\end{array}$ & \begin{tabular}{|l|}
$47,6214,50 b$ \\
$(26,12-65,51)$
\end{tabular} & 21,071 & $0,001 \psi^{* *}$ \\
\hline 4-HNE (ng/mL) & $\begin{array}{l}6,052,44 a \\
(1,81-9,921)\end{array}$ & $\begin{array}{l}58,8815,25 \mathrm{~b} \\
(39,93-83,32)\end{array}$ & \begin{tabular}{|l|}
$34,6114,91 b$ \\
$(11,77-55,59)$
\end{tabular} & 22,796 & $0,001 \psi^{* *}$ \\
\hline 80HdG (ng/mL) & $\begin{array}{l}13,864,59 a \\
(6,20-21,51)\end{array}$ & $\begin{array}{l}85,5110,46 b \\
(72,32-102,81)\end{array}$ & $\begin{array}{l}62,0120,93 b \\
(43,20-102,06)\end{array}$ & 21,621 & $0,001 \psi^{* *}$ \\
\hline PGF2 (ng/mL) & $\begin{array}{l}39,029,36 a \\
(22,07-57,71)\end{array}$ & $\begin{array}{l}217,5582,91 b \\
(122,11-392,02)\end{array}$ & $\begin{array}{l}102,2429,23 b \\
(63,21-163,10)\end{array}$ & 24,137 & $0,001 \psi^{* *}$ \\
\hline Net NO $(\mu \mathrm{M})$ & $\begin{array}{l}24,808,86 a \\
(14,53-40,56)\end{array}$ & $\begin{array}{l}55,7720,78 b \\
(31,33-95,16)\end{array}$ & $\begin{array}{l}32,5420,32 \mathrm{a} \\
(15,93-73,64)\end{array}$ & 11,052 & $0,004 \psi^{*}$ \\
\hline
\end{tabular}

${ }^{*} \mathrm{p}<0,05 * * \mathrm{p}<0,01$ 


\section{Discussion}

The formation of free radicals during abdominal surgery causes ischemia-reperfusion, leukocyte activation and mitochondrial dysfunction. Moreover, antioxidant levels decrease depending on the re-establishment of the stasis in the organism and biological depletion during the postoperative period. It is well known that laparoscopic surgery does not impair the immunological functions thanks to its non-invasive character and less induction of systemic inflammation. The increase in the intraabdominal pressure, decrease and increase of the intraabdominal swelling during the laparoscopy as a result of pneumoperitoneum may lead to ischemia-reperfusion and oxidative damage in the internal organs. It is also well known that hemodynamic changes and the effects of CO2 may cause oxidative stress during pneumoperitoneum [1,5]. In our study, in the rats, in which we formed experimental colorectal cancer, we compared the levels of the antioxidant molecules superoxide dismutase (SOD) and catalase (CAT) that are determinants of oxidative stress level caused by the laparoscopic colon resections. The levels of oxidative stress determinants and antioxidant molecules were higher in the subjects who underwent surgery compared to the control group. However, we found a statistically significant difference between the surgery groups. The levels of NT (ng/mL), 4-HNE (ng/mL), 8-OHdG $(\mathrm{ng} / \mathrm{mL})$, PGF2 $(\mathrm{ng} / \mathrm{mL})$, and Net NO $(\mu \mathrm{M})$ were higher in the laparotomy group compared to the laparoscopy group. This indicates that the impairment of hemostasis related to acute and chronic inflammation was most significant in the laparotomy group. M. Braga et al. conducted a study and found that the laparoscopic colorectal resection was related to a lower longterm complication incidence and higher quality of life compared to open surgery during the 12-month follow-up (6). We believed that this finding was related to the relatively lower release of oxidative stress determinants and antioxidant molecules after laparoscopic surgery. With the laparoscopic approach, postoperative complication rates and duration of hospitalization will decrease in patients who underwent colorectal cancer surgery. 8-hydroxy-2-desoxyguanosine (8-OHdG) is commonly defined as a determinant of oxidative stress.

In fact, this determinant is the most reliable marker of oxidative damage in DNA [3,7]. Pappas-Gogos et al. (2013) investigated the lipid and protein levels, RNA and nitrogen damage, 8-isoprostanes (8-epiPGF), 8-hydroxyguanosine (8$\mathrm{OHG}$ ) and 3-nitrotyrosine (3-NT) biomarkers in 60 randomized patients with colorectal cancer, who underwent laparoscopic or open surgery. The comparison of these parameters between the laparoscopy and open surgery groups showed that the levels of these parameters were significantly lower in the laparoscopy group even after 24 hours $[8,9]$. In our study, the 8-hydroxy-2desoxyguanosine $(8-\mathrm{OHdG})$ levels were significantly lower in the laparoscopy group compared to the laparotomy group. This finding indicated that patients with colorectal cancer would be exposed to less oxidative stress if the laparoscopic technique had been preferred.

Nowadays, advanced laparoscopy has become a widely used surgical procedure. As a result of the advantages such as the smaller incision compared to laparotomy, better visualization of the intraabdominal region, and shorter duration of surgery depending on the increase in experience, laparoscopy has become the gold standard in many surgical indications [10,11]. Although the trauma caused by the intervention is comparable between the laparoscopic and open surgery, the smaller trauma on the abdominal wall, shorter duration of surgery, early mobilization of patients, early oral feeding, shorter hospitalization, less pain, and less need for analgesics along with the decrease in the postoperative catabolic reactions are important improvements, which also enable the decrease of the treatment cost [12]. In addition to all these, stress during and after laparotomy may cause more destructive reactions during the postoperative period particularly in patients with malignant diseases. These complications may depend on the catabolic effect of the stress hormones released during laparotomy $[13,14]$.

\section{Study limitation}

Actual time planned for colon cancer was 16 weeks. When the rats died, the experiment was started at the 12 th week. The most important limitation of the study is that although the mass was seen in rats, not all of them were pathologically diagnosed with adenocarcinoma.

This study was scientifically supported as a Scientific Research Project by Aydın Adnan Menderes University (No: ADÜ BAP TPF-17052).

Disclosures: There is no conflict of interest for all authors.

Acknowledgements: None.

Funding: None.

\section{References}

1. Kopp I, Bauhofer A, Koller M. Understanding quality of life in patients with colorectal cancer: comparison of data from a randomised controlled trial, a population based cohort study and the norm reference population. Inflamm Res. 2004; 53:S130-5. https://oi. org/10.1007/s00011-004-0361-6

2. King PM, Blazeby JM, Ewings P, Kennedy RH. Detailed evaluation of functional recovery following laparoscopic or open surgery for colorectal cancer within an enhanced recovery programme. Int J Colorectal Dis. 2008; 23:795-800 https://doi.org/10.1007/s00384008-0478-0

3. Pappas-Gogos G, Tellis C, Lasithiotakis K, Tselepis AD, Tsimogiannis K, Tsimoyiannis E, Chalkiadakis G et al. Oxidative stress markers in laparoscopic versus open colectomy for cancer: a double-blind randomized study. Surg Endosc. 2013; 27(7):2357-2365 https://doi. org/10.1007/s00464-013-2788-8

4. Aspinen S, Harju J, Juvonen P, Selander T, Kokki H, Pulkki K et al. Scand J. Theplasma 8-OHdG levels and oxidative stress following cholecystectomy: a randomised multicentre study of patients with mini laparotomy cholecystectomy versus laparoscopic cholecystectomy. Scand j Gastroenterol 2016; 51(12):1507-1511 https://doi.org/10.1080/00365521.2016.1208270

5. Zulfikaroglu B, Koc M, Soran A, Isman FK, Cinel I. Evaluation of oxidative stress in laparoscopic cholecystectomy. SurgToday. 2002; 32(10): 869-874. https://doi.org/10.1007/s005950200169

6. Braga M, Frasson M, Vignali A, Zuliani W, Civelli V, et al. Laparoscopic vs. open colectomy in cancer patients: long-term complications, quality of life, and survival. Dis Colon Rectum. 2005; 48:2217-23 https://doi.org/10.1007/s10350-005-0185-7 
7. Glantzounis GK, Tselepis AD, Tambaki AP, Trikalinos TA, Manataki AD, Galaris DA et al.. Laparoscopic surgery-induced changes in oxidative stress markers in human plasma. Surg Endosc. 200; 15(11):1315-1319. https://doi.org/10.1007/s00464-001-0034-2

8. Stipancic I, Zarkovic N, Servis D, Sabolović S, Tatzber F, Busic Z. Oxidative stress markers afterlaparoscopicandopencholecystectomy. JLaparoendoscAdvSurgTech A. 2005; 15(4):347-352. https://doi.org/10.1089/lap.2005.15.347

9. Matsuda T, Fujita H, Kunimoto Y, Kimura T, OginoK.Analysis of Recurrent Casesafter Laparoscopic Surgery for Colorectal Cancer. Hepatogastroenterology. 2014; 61(132):1028-1032.

10. Arezzo A, Passera R, Ferri V, Gonella F, Cirocchi R, Morino M. Laparoscopic right colectomy reduces short-term mortality and morbidity. Results of a systematic review and meta-analysis. Int J Colorectal Dis. 2015; 30:1457-1472. https://doi.org/10.1007/s00384015-2304-9

11. Arung W, Drion P, Cheramy JP, Honore P, Meurisse M, Defraigne JO et al. Intraperitonealadhesionsafteropenorlaparoscopicabdominal procedure: an experimentalstudy in therat. J LaparoendoscAdvSurgTech A. 2012; 22(7):651-657. https://doi.org/10.1089/lap.2012.0102

12. Mynbaev OA, Eliseeva MY, Kalzhanov ZR, Lyutova L, Pismensky SV, Tinelli A, et al. Surgical trauma and CO2-insufflation impact on adhesion formation in parietal and visceral peritoneal lesions. Int J Clin Exp Med. 2013; 6(3):153-165.

13. Jurczok A, Schneider A, FornaraP.Inhibition of tumor implantationafter laparoscopy byspecificoligopeptides: a novelapproachtoadjuv antintraperitonealtherapytoprevent tumor implantation in an animal model. Eur Urol. 2007; 52(2):590-595. https://doi.org/10.1016/j. eururo.2006.10.057

14. KumarAS, Lee SW. Laparoscopy in Colorectal Surgery. Surg Clin Nort Am. 2013; 93:217-230. https://doi.org/10.1016/j.suc.2012.09.006 